This is the peer reviewed version of the following article: "Arias, F.J.; De Las Heras, S.A. (2019) Gas film heat transfer as enhancement strategy for phase change materials. Energy Storage, vol. 1, núm. 6: e95:1-e95:6." wich has been published in final form at [doi: 10.1002/est2.95]. This article may be used for non-commercial purposes in accordance with Wiley Terms and Conditions for Self-Archiving 


\title{
Gas Film Heat Transfer as Enhancement Strategy for Phase Change Materials
}

\author{
Francisco J. Arias* and Salvador De Las Heras ${ }^{a}$ \\ Department of Fluid Mechanics, University of Catalonia, \\ ESEIAAT C/ Colom 11, 08222 Barcelona, Spain
}

\begin{abstract}
In this work, consideration is given to an alternative strategy for phase change materials (PCMs) heat transfer enhancement with particular reference to horizontal plates. In contrast with current approaches in which heat transfer enhancement is pursued either by adapted geometries or acting on the thermal conductivity coefficient of a given PCM, here the problem is tackled by preventing the formation and contact of the front of solidification at the wall of the vessel by the deliberate presence of a gas film between the wall and the PCM. Because the presence of such a film, the front of solidification only can attains a certain critical thickness before gravitationally sinks by its own weight and then eliminating the continuous growth of the solid layer with the consequent reduction of conductive heat transfer and power output of the system as the solidification takes place which translates into an enhanced and steady power during the entire solidification process. It is shown that because the critical thickness of the solidified layer before sinks is a few millimeters or less and then smaller than practical gas films, therefore the heat transfer is controlled by the thickness and the thermal conductivity of the film. Additional $R \& D$ is required in order to arrive at a reliable practical and safe design.
\end{abstract}

Keywords. PCMs heat transfer enhancement, Thermal storage, Stationary power

\section{INTRODUCTION}

Despite advances made in phase change materials (PCMs) technology - including new techniques as well as new ad hoc materials with desired properties, nevertheless heat transfer enhancement will continue to play a crucial role in the future of phase change materials $(\mathrm{PCMs})$ for energetic applications. The low thermal conductivity (except the metallic-type) featured by almost all classes of pure PCMs - and specially the organic-type which are more attractive for domestic applications because the compatible range of temperatures, affects the rate of phase change process leading to a poor performance of the storage system. In order to mitigate the problem several techniques haven been proposed in the past years which roughly fall into two categories, namely. Those techniques of heat transfer enhancement based in adapted geometric configurations and extended surfaces; and those techniques in which heat transfer enhancement is attained by directly modifying in someway the thermal conductivity of the PCM. In the fist category we have, e.g, the use of of fins, heat pipes, or fillers [1], and in the second category we have the use of encapsulation [2] and [3]; or microencapsulation [4] and [5] of the PCM as well as the use of nanoparticles including nanotubes [6] or nanocomposites [7]. There are copious available literature of the topic and specially in the last advancements in PCMs, and for the interested reader on various techniques of heat transfer enhancement in latent heat thermal energy storage systems it is recommended the up-to-date review on the state-of-the-art as well as critical review [8].

In this work, consideration is given to an alternative strategy for phase change materials (PCMs) heat transfer enhancement with particular reference to horizontal plates in which the enhancement is attained neither by adapted geometries nor modification of the thermal heat transfer coefficients of the PCM but rather by preventing the formation, and contact of the front of solidification and the wall by the deliberate presence of a gas film covering the surface which limits the growth of the solidified layer to a certain critical thickness before gravitationally sinks by its own weight.

*Corresponding author: Tel.: +32 143321 94; Electronic address: francisco.javier.arias@upc.edu

This article has been accepted for publication and undergone full peer review but has not been through the copyediting, typesetting, pagination and proofreading process which may lead to differences between this version and the Version of Record. Please cite this article as doi: $10.1002 /$ est 2.95

This article is protected by copyright. All rights reserved. 


\section{MATERIALS AND METHODS}

For the sake of illustration, let us consider the most simple and illustrative case for a horizontal plate and restricted to one-dimensional and semi-infinite layer as depicted in Fig. 1. In order to obtain an analytical solution for the heat transfer, we will use the Stefan model which is traditionally used for preliminary estimations in PCMs analysis with the following assumptions [9]: changes in volume due to the solid-liquid phase change is neglected; heat is stored only as latent heat and sensible heat stored is negligible; heat transfer is only by conduction and there is not convection; when solidification starts, the PCM is liquid and its temperature is the phase change temperature, $T_{p c m}$; the temperature at the wall is $T_{o}$ and kept constant at this boundary for any later time.

With these simplifications, the heat flux per unit of area at the front of solidification driven by conduction is given by the well-known Fourier's law as

$$
q(t)=\frac{\kappa_{s} \Delta T}{s(t)}
$$

where $q$ is the heat flux per unit time; $t$ is the time of solidification; $\kappa_{s}$ is the thermal conductivity of the solidified layer; $\Delta T=T_{p c m}-T_{o}$ is the difference of temperature between the front of solidification where $T_{p c m}$ and the wall $T_{o}$; and $s$ is the thickness of the solidified layer. Referring to Eq.(1) it is easy to see that as the solidification front moves forward the power delivered by the PCM drops as $\propto s^{-1}$.

\section{A. solidified layer detachment}

The only way to control the thickness of the solidified layer as the solidification takes place is by the continuous removal of the solidified layer, and because it is desired to extract energy from the system (thermal energy) and not to provide energy, then the use of mechanical energy for detachment (e.g. vibrations, etc) of the solidified layer is discarded and therefore the only available force is gravity.

\section{Disjoining pressure}

In order to induce the detachment of the solidified layer there are certain pressures which are preventing the disjoining of the layer, and the pressure which must be applied to counterbalance these pressures is generally referred as the disjoining pressure, [10]. By looking at Fig. 1, the disjoining pressure $p$ must be equal or higher than the pressure pushing the solidified layer onto the wall, i.e.,

$$
p=p_{o}+\pi_{d}
$$

where $p_{o}$ is the pressure in the bulk or vessel (approximately atmospheric pressure for an unpressurized system), and $\pi_{d}$ is the adhesive resulting pressure resulting from attractive interaction forces between the solidified layer and the wall (Van der Waals forces) [11]. Classic theory predicts that the adhesive pressure for a thin liquid film on a flat surface is as follows [11]

$$
\pi_{d}=-\frac{A_{H}}{6 \pi \delta^{3}}
$$

where $A_{H}$ is the Hamaker constant, and $\delta$ is the liquid film thickness. The Hamaker constant is in the order of $A_{H} \approx 10^{-20} \mathrm{~J}$ and for the thickness $\delta$ around centimeters or less it is clear that $p_{o} \gg \pi_{d}$ and Eq.(2) becomes

$$
p \approx p_{o}
$$

Therefore, the pressure required for disjoining the solidified layer $p$ should be at least equal than the bulk pressure which for practical applications in PCMs is always the atmospheric pressure. On the other hand, the only force opposing this attachment is gravity, and this pressure is given by 


$$
p_{g}=\left(\rho_{s}-\rho_{l}\right) g s
$$

where $p_{g}$ is the gravitational downward pressure; $g$ is gravity; and $s$ is the thickness of the solidified layer. Therefore, by equating this pressure with the disjoining pressure, i.e., $p_{g}=p$ one obtains the critical thickness $s_{d}$ in which detachment occurs

$$
s_{d} \approx \frac{p_{o}}{\left(\rho_{s}-\rho_{l}\right) g}
$$

It is easy to see, that direct gravitational detachment is not possible. If one considers a change in density between the liquid and solid phase as $\rho_{s}-\rho_{l}=100 \mathrm{~kg} / \mathrm{m}^{3}$ at best (for water it is only $81 \mathrm{~kg} / \mathrm{m}^{3}$, and with the working pressure equal than atmosphere $p_{o} \approx 10^{5} \mathrm{~Pa}$, then it would require an unrealistic very thick layer before gravity can overcomes the bulk pressure.

\section{B. Film heat transfer}

Therefore, by our preceding discussion, we infer that in order to disjoin the solidified layer, the bulk pressure pushing the layer against the wall must be eliminated, and because depressurization is not an option, the only possibility is by equalizing the pressure in both sides (bottom and top) of the solidified layer and in this way the making the resulting force acting on the solidified layer identically zero. One way to do this is by using a film layer between the PCMs and the wall. This film, will allow not only that the solidified layer sinks almost immediately as is formed in the interface of the film-PCM but also as we will see, will control the entire process of heat transfer resulting into a stationary output power.

To begin with, let us consider the system depicted in Fig. 2 and Fig. 3. In this a heat transfer fluid (HTF) is circulating and extracting the latent heat from a PCM initially in liquid state which solidifies as the solidification process takes place. The HTF and the PCM are separated by a horizontal wall. Now, instead of allowing the formation and/or direct contact between the PCM and this wall ( as occurs in Fig. 1), a gas film layer is located in between which may be introduced by say, bubbling the gas from the bottom and being trapped in the top by buoyancy before the unity is sealed. The thickness of this film can be properly controlled by controlling the pressure of the system.

The object of this film, which must be as thin as possible, has the function to equalize the bulk pressure in both sides of the solidified layer, and then eliminating the bulk pressure which is opposing the gravitational detachment if the solidification occurs by direct contact with the wall. However, with the presence of the film a capillary force appears which could oppose the gravitational detachment. Nevertheless, this vertical surface tension force can dominate the hydrostatic pressure force only when the typical scale of the object is small compared to the so-called capillary length, $l_{c}$, which is given by [13]

$$
l_{c}=\left[\frac{\gamma_{A B}}{g\left(\rho_{B}-\rho_{A}\right)}\right]^{\frac{1}{2}}
$$

where $l_{c}$ is the the capillary length, $\gamma_{A B}$ the surface tension between the phases and $\rho_{A}$ and $\rho_{B}$ their densities. Therefore, one expects that dense objects may float at an interface provided that their typical size is less than this characteristic length. On this basis, capillary length for the solidified layer is given by

$$
l_{c} \approx\left[\frac{\gamma}{g\left(\rho_{s}-\rho_{l}\right)}\right]^{\frac{1}{2}}
$$

where $\rho_{s}$ and $\rho_{l}$ are the solid and liquid density of the PCM, respectively, and $\gamma$ the surface tension. For a PCM, $\left(\rho_{s}-\rho_{l}\right) \approx 80 \mathrm{~kg} / \mathrm{m}^{3}$ and if a high surface tension close to that of water $\gamma \approx 7 \times 10^{-2} \mathrm{~N} / \mathrm{m}$ is considered; we see that the capillary length is a few millimeters which is much less than the characteristic length expected of the solidified layer. Therefore, in view of the above argument, capillary forces preventing the sinking of the solidified layer can be neglected. 


\section{Attainable power using film heat transfer and gravitational detachment}

In this section we will derive an expression for the extractable power modified by the presence of the film.

To begin with, because the presence of the film, the total heat transfer between the PCM is not given by Eq.(1) but modified as [14]

$$
q_{f}(t)=\frac{\Delta T}{\frac{\delta_{f}}{\kappa_{f}}+\frac{s(t)}{\kappa_{s}}}
$$

where $\delta_{f}$ and $\kappa_{f}$ are the thickness and the thermal conductivity of the film, respectively. Because in absence of significant forces preventing the gravitational sinking of the solidified layer(as discussed previously), the sinking of the solidified layer starts almost at the same time it starts to be formed and from this point of view $\delta_{f} \gg s$. On the other hand the thermal conductivity of gases are equal or less than the PCM and then $\frac{\delta_{f}}{\kappa_{f}} \gg \frac{s(t)}{\kappa_{s}}$ and Eq. (9) becomes

$$
q_{f} \approx \frac{\kappa_{f} \Delta T}{\delta_{f}}
$$

which means that the heat transfer is controlled by the film.

Finally, it is interesting to compare the stationary power predicted by Eq.(10) using a film layer and the power from traditional approaches, i.e., by direct contact of the solidified layer with the wall and its continuous growth given by Eq.(1). By dividing Eq.(10) with Eq.(1) one obtains the ratio between the density powers as

$$
\frac{q_{f}}{q}=\frac{\kappa_{f}}{\kappa_{s}} \frac{s(t)}{\delta_{f}}
$$

which is a function of time.

\section{DISCUSSION AND RESULTS}

To obtain some idea of the power enhancement predicted by Eq.(11), we assume some typical values of the parameters: A helium film with $\kappa_{f}=0.15 \mathrm{~W} /(\mathrm{m} \mathrm{K})$ at room temperature ( given the chemical inactivity of helium makes this gas specially attractive for this purpose if it is considered that it must be in direct contact with the $\mathrm{PCM})$ and using as PCM a paraffine wax $\kappa_{s}=0.2 \mathrm{~W} /(\mathrm{m} \mathrm{K})$ and water $\kappa_{s}=0.5 \mathrm{~W} /(\mathrm{m} \mathrm{K})$ as representative for organic and inorganic PCMs, respectively. The resulting curves are shown in Fig 4. It is seen that for the case of organic PCMs which are the most suitable for domestic applications owing to their range of melting temperatures, the power delivered by the use of helium film will start to be higher from $\frac{s}{\delta_{f}}>1.2$ and when $\frac{s}{\delta_{f}} \approx 12$ the power delivered by the film will be almost 10-fold in comparison with the direct contact PCM-wall. In this contest, for example, the power delivered by a horizontal plate when the front of solidification is $5 \mathrm{~cm}$ thickness will be one tenth of the power delivered if the all is covered by a helium film with a thickness of $0.5 \mathrm{~cm}$, which is a considerable enhancement caused by the film. The use of of gas films open the possibility to obtain both a constant power through the entire process of solidification and also a much larger power density which translates into compact devices.

As regard the possible limitations of the proposed film gap method, the main of them is related with the limitation to horizontal heat transfer surfaces and with PCMs whose density decreases with temperature. The first limitation is necessary if simple and robust designs are desired in which the gas film is formed and trapped by buoyancy between the wall and the PCM when it is molten, and the second requirement to induce gravitational sinking of the solidified layer by its own weight as was pictorially depicted in Fig. 3. The first requirement can be easily accomplished by using horizontal plates and for the second fortunately the most interesting PCMs for domestic applications -i.e., organic PCMs, their density decrease with the temperature. Concerning the introduction of a gas film, there is not appreciable technical problem in introducing such a film between the wall and the PCM, e.g. a possible technique of fabrication could be the injection of the inert gas by bubbling the gas from the bottom when the PCM is molten and then by buoyancy it will will rise covering the top surface. The control of the thickness of the film can be made easily by controlling the pressure of the system once the gas is introduced and the system is sealed. 


\section{SUMMARY OF RESULTS AND CONCLUSIONS}

A novel strategy for phase change materials (PCMs) heat transfer enhancement was outlined. The idea lies in preventing the formation and attachment of the front of solidification to the wall by the deliberate presence of a gap filled with an inert gas favoring the gravitational falling of the solidified layer once its critical weight is attained. Some interesting conclusions are derived from this preliminary work as follows:

(a) Heat transfer through a gas film eliminates the progressive reduction of power output which occurs in traditional system as the solidification takes place and provides a stationary power during the entire solidification of the PCM.

(b) Because the thickness of the film is much larger than the critical thickness of the solidified layer, then the heat transfer process is controlled by the film.

(c) The power enhancement using a film is larger inasmuch the solidification progress.

(d) Additional $\mathrm{R} \& \mathrm{D}$ is required in order to arrive at a reliable practical and safe design.

\section{Nomenclature}

$A_{H}=$ Hamaker constant

$g=$ gravity

$h=$ change of enthalpy of solidification

$l_{c}=$ capillary length

$p=$ pressure

$s=$ distance surface- front of solidification

$t=$ time

$\Delta T=$ temperature difference

\section{Greek symbols \\ $\rho=$ density \\ $\kappa=$ thermal conductivity \\ $\delta=$ film thickness \\ $\Delta=$ difference \\ $\pi=$ adhesive pressure}

\section{subscripts symbols}

$o=$ atmospheric

$f=$ film

$l=$ liquid

$s=$ solid

$g=$ gravitational

\section{Acknowledgements}

This research was supported by the Spanish Ministry of Economy and Competitiveness under fellowship grant Ramon y Cajal: RYC-2013-13459.

\section{REFERENCES}

[1] Huang, J, Dai, J, Peng, S, Wang, T, Hong, S. 2019. Modification on hydrated salt-based phase change composites with carbon fillers for electronic thermal management. Int J Energy Res. 43: 3550-3560.

[2] Hawlader, M. N., Uddin, M. S. and Zhu, H. J. 2002. Encapsulated phase change materials for thermal energy storage: Experiments and simulation. Int. J. Energy Res., 26: 159-171. 
[3] Tabrizi, N. and Sadrameli, M. 2003, Modelling and simulation of cyclic thermal regenerators utilizing encapsulated phase change materials (PCMs). Int. J. Energy Res. 27: 431-440.

[4] Beyhan, B., Cellat, K., Konuklu, Y., Gungor, C., Karahan, O., Dundar, C., and Paksoy, H. 2017. Robust microencapsulated phase change materials in concrete mixes for sustainable buildings. Int. J. Energy Res., 41: 113-126.

[5] Konuklu, Y, Erzin, F. 2019. Preparation of pentadecane/poly(melamine-urea-formaldehyde) microcapsules for thermal energy storage applications. Int J Energy Res. 1-5.

[6] Yang, L, Zhang, N, Yuan, Y, Cao, X, Xiang, B. Thermal performance of stearic acid/carbon nanotube composite phase change materials for energy storage prepared by ball milling. Int J Energy Res. 2018; 1-10.

[7] Xu, X, Zhang, X, Liu, S. 2018. Experimental study on cold storage box with nanocomposite phase change material and vacuum insulation panel. Int J Energy Res. 42: 4429-4438.

[8] Ibrahim N.I., Al-Sulaiman F.A., Rahman S., Yilbas B.S., Sahin A.Z. 2017.Heat transfer enhancement of phase change materials for thermal energy storage applications: A critical review. Renewable and Sustainable Energy Reviews. 74, p.p. 26-50

[9] Mehling H., Cabeza L.F. 2008. Heat and cold storage with PCM An up to date introduction into basics and applications. Springer.

[10] Hans-Jurgen Butt, Karlheinz Graf, Michael Kappl. Physics and chemistry of interfaces. John Wiley \& Sons Canada, Ltd., 1 edition, 2003

[11] Israelachvili J.N. 2011. Intermolecular and Surface Forces. Academic Press, Revised Third edition. ISBN: 9780123919274.

[12] Keller J.B. 1998. Surface tension force on a partly submerged body. Physics of Fluids. 10, (11). pp. 3009-3010.

[13] Robert Vella D.J.2007 The Fluid Mechanics of Floating and Sinking. PhD thesis, University of Cambridge, August 2007

[14] Kreith F; manglik R.M; Bohn M.S. 2011. Principles of Heat Transfer. Seveth edition. SI. CENGAGE Learning 


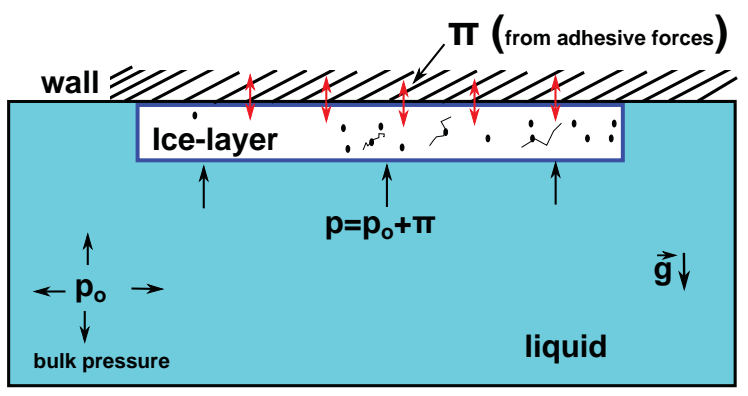

FIG. 1: Disjoining pressures surrounding a growing solidified layer. 

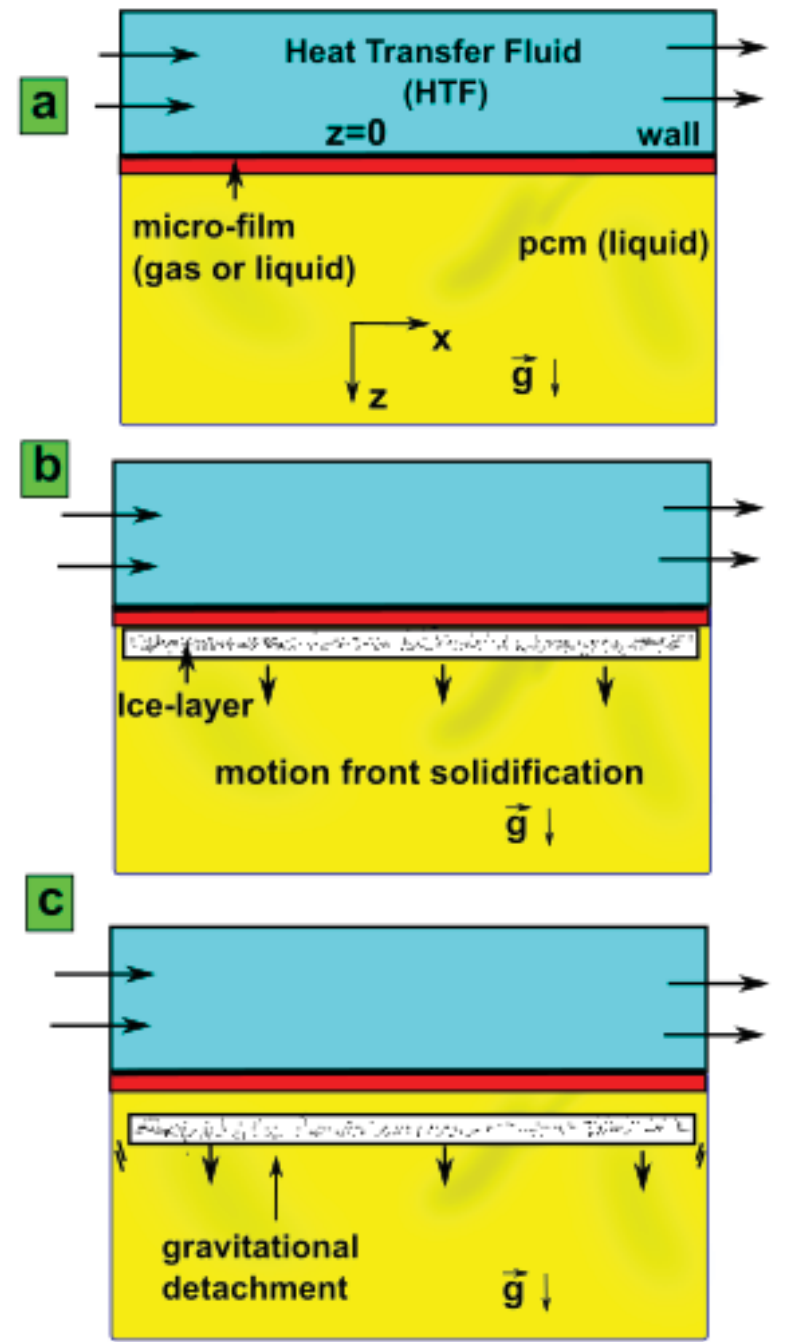

FIG. 2: Sketch of the sequence for film heat transfer gravitational detachment from a horizontal surface.

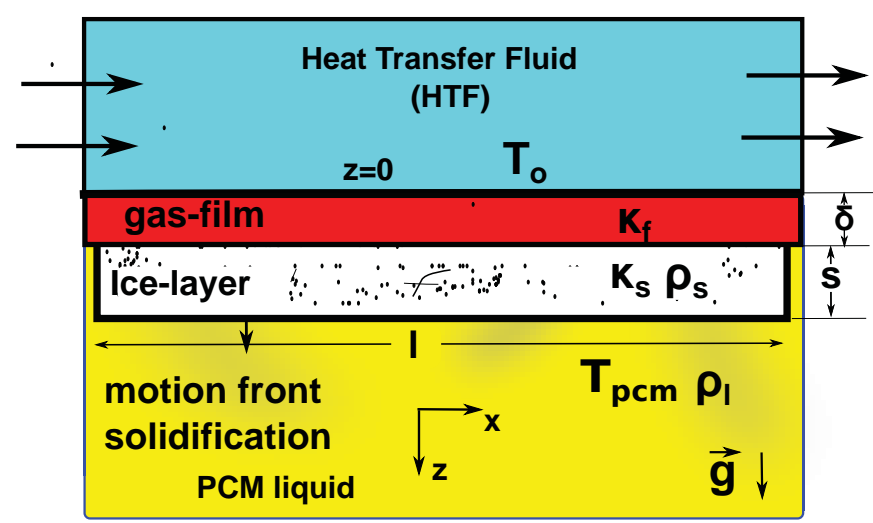

FIG. 3: Physical model for film heat transfer gravitational detachment from a horizontal surface. 
